\title{
The impact of the COVID-19 pandemic on cancer
} care

\author{
The COVID-19 pandemic has disrupted the spectrum of cancer care, including delaying diagnoses and treatment \\ and halting clinical trials. In response, healthcare systems are rapidly reorganizing cancer services to ensure that \\ patients continue to receive essential care while minimizing exposure to SARS-CoV-2 infection.
}

Mike Richards, Michael Anderson, Paul Carter, Benjamin L. Ebert and Elias Mossialos

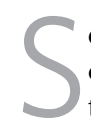
evere acute respiratory syndrome coronavirus 2 (SARS-CoV-2), the causative virus of coronavirus disease 2019 (COVID-19), continues to spread globally at an alarming rate. The unprecedented burden of COVID-19 on health systems worldwide has important implications for cancer care. First, although the data remain limited, patients with cancer appear to be more vulnerable to worse outcomes from the infection, including greater need for ventilator support ${ }^{1}$ and elevated mortality rates ${ }^{2,3}$. Second, diagnosis may be delayed as screening programs and diagnostic services have been decreased or suspended in many countries, and patients, wary of exposing themselves to the risk of infection, have been more reluctant to present to healthcare services. Third, treatment pathways have been altered to minimize potential exposure of patients with cancer to SARS-CoV-2 and to reduce the risk during surgery or radiation therapy. Fourth, certain aspects of ongoing care have been deprioritized to enable health systems to respond to the COVID-19 pandemic, which has resulted in patients' receiving suboptimal or delayed care. Fifth, many clinical trials have been suspended, which has reduced current therapy options for patients who might have participated and has jeopardized longer-term therapy development. In response, healthcare professionals and managers in many countries have acted quickly to mitigate the repercussions of COVID-19 on the provision of cancer care by reorganizing cancer services and updating guidance for medical staff and patients. Here we consider these developments throughout the patient pathway, from diagnosis to treatment and ongoing care.

\section{Implications for diagnosis}

The necessity to divert healthcare staff and resources to address the pandemic has resulted in the suspension of cancer screening programs for asymptomatic patients in many countries. In March 2020, the Welsh government (https://phw.nhs. wales/news/novel-coronavirus-covid-19temporarily-pauses-some-of-the-screeningprogrammes-in-wales/) and the Scottish government (https://www.gov.scot/news/ health-screening-programmes-paused/) suspended screening programs for breast, cervical and bowel cancer. In April, the Northern Ireland government followed (https://www.health-ni.gov.uk/news/ temporary-pause-routine-screeningprogrammes), with England yet to formally announce they are suspending screening. In the USA, the Centers for Medicare \& Medicaid Services have classified screening as a low-priority service and suggested healthcare organizations consider postponing screenings ${ }^{4}$. In addition, many patients have been fearful of exposure to SARS-CoV-2 or of overburdening healthcare services and thus have been less likely to present to healthcare services for cancer screening and diagnosis. As an example, emergency-department visits in England dropped by nearly a third in March 2020 compared with the same month the previous year (https://www.england. nhs.uk/statistics/statistical-work-areas/ ae-waiting-times-and-activity/). As approximately one in five cancers are diagnosed in emergency presentations (https://www.cancerdata.nhs.uk/ routestodiagnosis/routes), this is likely to be responsible for considerably delayed diagnoses. In addition, the interim Chief Medical Officer for Scotland reported that urgent referrals of patients with cancer by primary-care physicians had been reduced by over $70 \%$ by mid-April compared with the weekly average over the past 3 years (https://www.bbc.co.uk/ news/uk-scotland-52353657). Similar reductions have been reported in England ${ }^{5}$. By assuming urgent cancer referrals have a conversion rate of 7\%, Cancer Research UK has estimated that this reduction in referrals could mean around 2,000 fewer cancers are being diagnosed per week ${ }^{5}$.
Most forms of endoscopy, but particularly upper procedures, are classified as aerosol generating, which increases the risk of SARS-CoV-2 transmission, as also noted in the guidance of the British Society of Gastroenterology (https:// www.bsg.org.uk/covid-19-advice/ endoscopy-activity-and-covid19-bsg-and-jag-guidance/). Colonoscopies are also risk prone, due to prolonged fecal shedding of the virus ${ }^{6}$. Thus, there has been consensus among the American College of Gastroenterology (https://gi.org/2020/03/15/ joint-gi-society-message-on-covid-19/), the European Society of Gastrointestinal Endoscopy (https://www.esge.com/assets/ downloads/pdfs/general/ESGE_ESGENA_ Position_Statement_gastrointestinal_ endoscopy_COVID_19_pandemic.pdf), and the Asian Pacific Society for Digestive Endoscopy that elective endoscopies should be suspended ${ }^{7}$. As a result, delivery of endoscopy services has been markedly decreased. For example, in the UK, the number of endoscopies undertaken were reduced by over $90 \%$ in April 2020 compared with the first 3 months of 2020 , based on data from the UK National Endoscopy Database (https://ned.jets.nhs. $\mathrm{uk} / \mathrm{KPI} /$ ). It should be noted that as different countries pass their peak of COVID-19 cases, such recommendations are being reconsidered. In the meantime, demand for non-invasive imaging, such as computed tomography, has increased, as it carries a lower infection risk. To limit the need for prolonged deep cleaning of equipment after scanning of patients with COVID-19 and to decrease the risk of exposing other patients to infection, many hospitals are using separate COVID-19-exposed and non-exposed scanners. Moving forward, the continuation of diagnostic services, including endoscopy, may be facilitated by the setting up of diagnostic hubs that are kept as free as possible from SARS-CoV-2 exposure by being located in designated sites with extensive capacity for COVID-19 
testing of patients and staff, and mandating self-isolation of patients for 7-14 days before attendance.

\section{Implications for surgery}

Capacity for surgery has also been reduced as theater space and ventilators have been requisitioned to provide additional critical care capacity for patients with COVID-19. Moreover, anecdotal reports of patients developing COVID-19 after admission to hospital for elective surgery have resulted in patients' being more reluctant to have surgery. As a result, many cancer centers have been using neoadjuvant chemotherapy, radiotherapy or hormonal therapy as therapeutic modalities while surgeries are being delayed. However, these are difficult decisions that involve a risk of disease progression or emergency admissions with complications. To aid clinicians in navigating this complex decision-making process, relevant clinical societies and associations in many countries have issued guidance on this. For instance, the American College of Surgeons has recommended that in the early stages of the pandemic, semi-elective surgery, such as for nearly obstructing colon cancers, stenting for esophageal cancers and most gynecological cancers, should continue (https://www. facs.org/covid-19/clinical-guidance/ elective-case). They recommend that around the peak of the pandemic, only urgent or emergent surgery, such as for perforated, obstructed or actively bleeding cancers, should continue. The National Health System in England (NHS England) has recommended that at the peak of the pandemic, hospitals should prioritize patients who require an urgent operation within the subsequent 24-72 hours ${ }^{8}$.

As a direct consequence of these changes, services are being reorganized to address a growing backlog of semi-elective cancer operations in many countries. NHS England is consolidating cancer surgery in cancer hubs kept as free as possible from SARS-CoV-2 exposure ${ }^{8}$. This echoes similar developments in Lombardy, Italy ${ }^{9}$. Based on the models set out in these countries, such COVID-19-free facilities should be ideally located in sites separate from acute-care hospitals. If designating geographically distinct COVID-19 facilities and cancer-care facilities is not possible, separate facilities should be designated in mixed-care sites, with dedicated access and admissions processes for patients with cancer. In addition, services should be delivered by a designated pool of staff to reduce the risk of exposing SARS-CoV-2-free patients and healthcare workers to the virus. Patients should be screened with strict rules of admitting only patients with no symptoms suggestive of COVID-19 who have self-isolated for 7-14 days and have tested negative for SARS-CoV-2. As capacity builds in such facilities, it would be important to develop a program of staff testing and of triaging the backlog of unmet demand for cancer surgery on the basis of clinical need. This will require active monitoring of waiting lists and engagement with patients remotely to update their priority status.

\section{Implications for radiotherapy}

Radiotherapy has always had a major role in cancer both as a curative therapy and as a palliative therapy. This role has expanded with COVID-19, as radiation therapy has often been used to replace or to delay other treatment modalities with higher infection risk $^{10}$. For example, an international panel of experts has recommended that a short course of radiotherapy can be used to delay surgery in patients with rectal cancer ${ }^{10}$. Reports from China and Italy have indicated that strict infection prevention and control measures, including the screening and testing of patients, can allow radiotherapy services to continue relatively undisrupted throughout the pandemic ${ }^{11,12}$. However, as demand increases, courses of radiotherapy may need to be rationed. Both the National Institute for Health and Care Excellence in the UK (https://www.nice.org.uk/guidance/ ng162) and the American Society of Radiation Oncology in the USA (https:// www.astro.org/Daily-Practice/COVID19-Recommendations-and-Information/ Clinical-Guidance) have issued guidance that recommends prioritizing radiation therapy for patients with rapidly proliferating tumors, such as head and neck cancers. Lower priority indications for radiotherapy include adjuvant radiotherapy in resected disease, and radical radiotherapy for prostate cancer in patients having neo-adjuvant hormone therapy. In addition, to minimize exposure of patients to infection, hypofractionation regimens are being used ${ }^{13}$.

\section{Implications for systemic treatment}

Systemic treatment options such as chemotherapy may expose patients to a higher risk of becoming infected and may lead to worse outcomes once they develop COVID-19 ${ }^{14}$. The risk associated with biologic and monoclonal-antibody therapies is less clear, as some of them may be beneficial in combating the inflammatory storm seen in COVID-19 ${ }^{15}$. For example, clinical trials are currently underway to assess the effectiveness of tocilizumab (https://clinicaltrials.gov/ ct2/show/NCT04317092) and baricitinib (https://clinicaltrials.gov/ct2/show/ NCT04320277) in treating COVID-19. The European Society for Medical Oncology (https://www.esmo.org/guidelines/ cancer-patient-management-during-thecovid-19-pandemic) and the American Society of Clinical Oncology (https://www. asco.org/asco-coronavirus-information/ care-individuals-cancer-during-covid-19) have published several guidelines to inform the systemic treatment of cancer in the current circumstances. When chemotherapy is associated with dramatic improvements in outcomes, as is the case for many acute hematological malignancies or extensive small-cell lung cancer, there is consensus that the provision of chemotherapy must continue, although viral testing should be performed before therapy. When the absolute benefit of chemotherapy may be marginal, such as in the case of palliative or adjuvant chemotherapy for elderly and comorbid patients, then treatment may be postponed indefinitely or alternative treatment options, such as hormonal therapy, should be considered. These difficult conversations that clinicians need to have with patients are complicated by considerable uncertainty about the risks and benefits associated with each treatment option while the pandemic is ongoing.

\section{Implications for ongoing care}

The recommendations of the European Society for Medical Oncology for the management and treatment of cancer in the COVID-19 era have classified follow-up for most cancers, depending on risk of relapse, as either low or medium priority under the current circumstances. However, this reprioritization may have long-term implications for the detection of disease progression and complications that may impact cancer outcomes. In addition, the ability of primary-care providers to offer face-to-face appointments has been reduced, with routine services in many countries being switched to tele-consultations unless an in-person consultation is deemed essential. Although these changes are important in maintaining a level of continuity of care, they may be reducing opportunities for detecting symptoms of recurrence, undertaking advanced-care planning or coordinating palliative care. In addition, the psychological stress of receiving a diagnosis and treatment for cancer will be exacerbated in current circumstances, as many patients may struggle to access their usual support networks, and the provision of psychological support through healthcare systems may be reduced. Survivorship and psychological support services are adapting to provide 
services remotely when possible. However, there is a risk of digital exclusion for those who lack routine internet access and for elderly and comorbid populations, who are known to have lower levels of digital literacy.

\section{Implications for clinical trials}

Apart from diagnosis, treatment and care, COVID-19 has resulted in the suspension of many clinical trials, for at least the peak of the pandemic. There are currently over 12,000 active clinical trials in the area of cancer (https://www.centerwatch.com/ clinical-trials/listings/therapeutic-area/12/ oncology/), and halting many of them will have long-lasting health and financial consequences. Beyond the loss of the potential health benefits for patients already enrolled in the trials, the cost of an oncology phase 3 clinical trial is on average at least $\$ 20$ million $^{16}$, which could pose a considerable financial hurdle in replanning suspended trials in the future and could jeopardize the pipeline of the identification and approval of new cancer treatments. Ideally, clinical trials should not be suspended unless a thorough and rapid risk assessment has taken place. The US Food and Drug Administration ${ }^{17}$ and the European Medicines Agency ${ }^{18}$ have both issued guidance on this topic. Specifically, the former recommends that ongoing use of the investigational product be context dependent and take account of the nature of the investigational product, the ability to conduct appropriate safety monitoring and the nature of the disease under study in the trial ${ }^{17}$. Pragmatic steps that could minimize disruption to clinical trials include the conversion of physical visits to phone or video visits, and transferring study participants to other active sites of cancer care if use of the primary site is disrupted. However, transferring trial participants needs to be justified on the individual risk/ benefit ratio of each study participant.

\section{Conclusions}

Given the unprecedented effects of COVID-19 on health systems worldwide, it is unfortunately inevitable that this pandemic will have a substantial impact on cancer outcomes ${ }^{1-3}$. Beyond the risks that direct acquisition of the virus may carry for patients with cancer, delayed diagnosis and the provision of suboptimal care may have a larger impact for the wider population of patients with cancer. It is imperative that healthcare organizations, clinical oncology associations and relevant regulatory bodies take action to mitigate the negative impact on cancer outcomes. In the short term, diagnostic and treatment pathways need to be adapted to minimize exposure to and poorer outcomes from SARS-CoV-2 infection. Once such measures have been implemented successfully, it would be equally important to reassure the public that it is safe to seek medical advice for symptoms. In the medium term, cancer services need to be reorganized to protect patients with cancer and allow the resumption of cancer surgery and endoscopy. This will involve the use of designated cancer hubs and strict infection prevention and control policies. In the long term, the capacity to undertake diagnostics and elective surgery needs to be expanded to address a growing backlog of delayed diagnosis and unmet needs. The impact of the COVID-19 pandemic on the treatment and care of patients who do not have COVID-19 should not be understated. However, negative outcomes can be minimized through the concerted efforts of healthcare providers and regulatory bodies, individual clinicians and patients.

\section{Mike Richards ${ }^{1}$, Michael Anderson (D)2 Paul Carter ${ }^{3}$, Benjamin L. Ebert (iD ${ }^{4}$ and Elias Mossialos ${ }^{5,6}$ 四 \\ ${ }^{1}$ The Health Foundation, London, UK. ${ }^{2}$ Department} of Health Policy, London School of Economics and
Political Science, London, UK. ${ }^{3}$ Department of Public Health and Primary Care, University of Cambridge, Cambridge, UK. ${ }^{4}$ Brigham and Women's Hospital Dana-Farber Cancer Institute, Harvard Medical School, Boston, USA. ${ }^{5}$ Department of Health Policy, London School of Economics and Political Science, London, UK. ${ }^{6}$ Institute of Global Health Innovation, Imperial College London, London, UK.

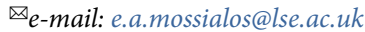

Published online: 20 May 2020

https://doi.org/10.1038/s43018-020-0074-y

References

1. Liang, W. et al. Lancet Oncol. 21, 335-337 (2020).

2. Wu, Z. \& McGoogan, J. M. JAMA 323, 1239-1242 (2020).

3. Onder, G., Rezza, G. \& Brusaferro, S. JAMA 323, 1775-1776 (2020).

4. Centers for Medicare \& Medicaid Services. https://www.cms.gov/files/document/ cms-non-emergent-elective-medical-recommendations. pdf (2020).

5. Cancer Research UK. https://www.cancerresearchuk.org/sites/ default/files/april2020 cruk_hsc_submission_covid_cancer final_public.pdf (2020).

6. Wu, Y. et al. Lancet Gastroenterol. Hepatol. 5, 434-435 (2020).

7. Chiu, P.W.Y. et al. Gut https://doi.org/10.1136/gutjnl-2020321185 (2020).

8. NHS England. https://www.england.nhs.uk/coronavirus/wpcontent/uploads/sites/52/2020/04/C0239-Specialty-guideEssential-Cancer-surgery-and-coronavirus-v1-70420.pdf (2020).

9. Curigliano, G. ASCO Daily News https://dailynews.ascopubs.org/ do/10.1200/ADN.20.200068/full/ (2020).

10. Marijnen, C.A.M. et al. Radiother. Oncol. https://doi. org/10.1016/j.radonc.2020.03.039 (2020).

11. Xie, C. et al. medRxiv https://doi. org $/ 10.1101 / 2020.03 .21 .20037051$ (2020).

12. Krengli, M., Ferrara, E., Mastroleo, F., Brambilla, M. \& Ricardi, U. Adv. Radiat. Oncol. https://doi.org/10.1016/j.adro.2020.03.003 (2020).

13. Yahalom, J. et al. Blood https://doi.org/10.1182/blood.2020006028 (2020).

14. Zhang, L. et al. Ann. Oncol. https://doi.org/10.1016/j. annonc.2020.03.296 (2020).

15. Stebbing, J. et al. Lancet Infect Dis. https://doi.org/10.1016/S14733099(20)30132-8 (2020).

16. Sertkaya, A., Wong, H.-H., Jessup, A. \& Beleche, T. Clinical Trials https://doi.org/10.1177/1740774515625964 (2016).

17. US Food and Drug Administration. https://www.fda.gov/ media/136238/download (2020).

18. European Medicines Agency. https://ec.europa.eu/health/sites/ health/files/files/eudralex/vol-10/guidanceclinicaltrials_covid19 en.pdf (2020).

Competing interests

The authors declare no competing interests. 